\title{
Open problems in Gaussian fluid queueing theory
}

\author{
K. Dȩbicki • M. Mandjes
}

Received: 9 May 2011 / Revised: 9 May 2011 / Published online: 6 July 2011

(C) The Author(s) 2011. This article is published with open access at Springerlink.com

\begin{abstract}
We present three challenging open problems that originate from the analysis of the asymptotic behavior of Gaussian fluid queueing models. In particular, we address the problem of characterizing the correlation structure of the stationary buffer content process, the speed of convergence to stationarity, and analysis of an asymptotic constant associated with the stationary buffer content distribution (the so-called Pickands constant).
\end{abstract}

Keywords Buffer content process · Fractional Brownian motion · Gaussian processes - Queues

Mathematics Subject Classification (2000) Primary 60G15 · Secondary 60G70

\section{Introduction}

Over the last years a substantial effort has been devoted to the analysis of queueing fluid systems driven by Gaussian processes; see the monograph [16] and references

K. Dębicki (凶)

Mathematical Institute, University of Wrocław, pl. Grunwaldzki 2/4, 50-384 Wrocław, Poland e-mail: Krzysztof.Debicki@math.uni.wroc.pl

M. Mandjes

Korteweg-de Vries Institute for Mathematics, University of Amsterdam, Amsterdam, The Netherlands

e-mail: M.R.H.Mandjes@uva.nl

M. Mandjes

Eurandom, Eindhoven University of Technology, Eindhoven, The Netherlands

M. Mandjes

CWI, Amsterdam, The Netherlands 
therein. On the one hand, the interest in such models stems from both the flexibility and richness of the family of Gaussian processes; more specifically, Gaussian processes cover a broad spectrum of correlation structures, which for instance cover phenomena as long-range dependence and self-similarity. On the other hand, both empirical and theoretical considerations legitimate the use of Gaussian processes as models for traffic streams in modern communication networks. The empirical evidence consists of a variety of measurement studies that statistically assess the properties of network traffic. As a theoretical back-up we mention in particular [7, 15], which proved that in a heavy traffic environment parameterization, large numbers of i.i.d. on-off sources may be approximated by a Gaussian process (with the same covariance structure as the on-off process). Additionally, contributions by Taqqu et al. [26] and Mikosch et al. [20] gave a formal argument for the use of a specific Gaussian process, viz. fractional Brownian motion. In [5, 6] it was proved that these central limit theorem type of results carry over to the buffer content process level. This formally justified the analysis of buffer content processes for queues fed by Gaussian input.

Let $\{Q(t): t \geq 0\}$ be the stationary buffer content process for a fluid queue fed by a centered Gaussian stochastic process $\{X(t): t \in \mathbb{R}\}$ with stationary increments, continuous sample paths a.s. and variance function $\sigma_{X}^{2}(t)=\operatorname{Var}(X(t))$. We assume that the system is emptied with a constant output rate $c>0$. Due to Reich [23], the following representation for $Q(t)$ holds on the process level:

$$
\{Q(t): t \geq 0\}=_{\mathrm{d}}\left\{\sup _{s \leq t}(X(t)-X(s)-c(t-s)): t \geq 0\right\}
$$

with the interpretation that $A(s, t)=X(t)-X(s)$ is the amount of input that entered the system in time interval $[s, t), s<t$. For notational convenience we write $Q$ instead of $Q(t)$, if one dimensional properties of the queueing process are analyzed (in stationarity).

The following special cases of $X(\cdot)$ play a crucial role in the literature:

- The case of fractional Brownian motion (FBM): $X(t)=B_{H}(t)$, where $B_{H}(\cdot)$ is a fractional Brownian motion with Hurst parameter $H \in[1 / 2,1)$; that is, $X(\cdot)$ is a centered Gaussian process with stationary increments, continuous sample paths, $X(0)=0$ a.s., and variance function $\sigma_{X}^{2}(t)=t^{2 H}$; see, e.g., $[5,13,16,21]$.

- The case of Integrated Gaussian (IG) processes: $X(t)=\int_{0}^{t} Z(s) \mathrm{d} s$, where $Z(\cdot)$ is a centered stationary Gaussian process with continuous covariance function $R(t)=$ $\operatorname{Cov}(Z(s+t), Z(s))$; see, for example, $[6,15,16]$.

Both classes of inputs possess the property that $\sigma_{X}^{2}(t)$ is regularly varying at $\infty$ with index $\alpha_{\infty} \in[1,2)$. We refer to the monograph [16] for a complete survey on Gaussian queueing models.

In this paper we present three problems in Gaussian fluid model theory. Despite the substantial research efforts devoted, these problems are still open, as far as we are aware. 


\section{Correlation structure of Gaussian queue}

For Gaussian queues, so far the focus was on the characterizing the steady-state distribution of $\{Q(t): t \geq 0\}$. Much less is known about the dependence structure of the queueing process, represented by the correlation function:

$$
\rho(t):=\operatorname{Corr}(Q(t), Q(0))=\operatorname{Cov}(Q(t), Q(0)) / \operatorname{Var}(Q(0)) .
$$

Properties of $\rho(t)$ have been studied for several other queueing systems; see [12, 24] and references therein. It is the dependence structure of the Gaussian input process that makes standard techniques not applicable. An additional difficulty in analyzing the asymptotics of $\rho(t)$ is that for a general Gaussian input process $X(\cdot)$, there are no explicit expressions available for the (stationary) distribution of $Q$, let alone of the transient $Q(t)$.

The relevance of insight into properties of $\rho(t)$ (as $t \rightarrow \infty)$ is evident, not only in view of engineering purposes. From a more general standpoint, an interesting and important fundamental question can be stated: is the short/long-range dependence property on the level of input process $X(\cdot)$ inherited by the workload process? Apart from the case of the Brownian queue (also referred to as reflected Brownian motion), for which $\rho(t)$ decays exponentially fast to 0 (see [1]), the answer to this question is open. Interestingly, it is anticipated that for $\{X(t)\}$ being long-range dependent, the asymptotics of $\rho(t)$ are decaying polynomially, equally fast as the asymptotics of $\operatorname{Cov}(X(1), X(t+1)-X(t))[18]$. More precisely, we expect the following.

\section{Conjecture 2.1}

(i) If $\alpha_{\infty}=1$, then for some constant $\gamma_{1} \in(0, \infty)$, as $t \rightarrow \infty$,

$$
\lim _{t \rightarrow \infty} \frac{\log \rho(t)}{t}=-\gamma_{1}
$$

(ii) If $\alpha_{\infty} \in(1,2)$, then for some constant $\gamma_{\alpha_{\infty}} \in(0, \infty)$, as $t \rightarrow \infty$,

$$
\lim _{t \rightarrow \infty} \frac{\rho(t)}{\sigma_{X}^{2}(t) / t^{2}}=\gamma_{\alpha_{\infty}} .
$$

The above conjectures are to some extent supported by findings in $[9,11]$, where the asymptotics of $\mathbb{P}\left(Q(0)>u, Q\left(t_{u}\right)>u\right)$, as $u \rightarrow \infty$ were derived for various classes of functions $t_{u}$.

\section{Speed of convergence to stationarity}

Assuming that at time 0 the system is empty, the transient buffer content $Q_{\mathrm{tr}}(t)$ at time $t>0$ obeys $Q_{\operatorname{tr}}(t)={ }_{\mathrm{d}} \sup _{s \in[0, t]}(X(s)-c s)$. Knowledge of the speed of convergence of $Q_{\mathrm{tr}}(t)$ to $Q$ is intimately related to many aspects of the analyzed queueing system. For instance, it helps to determine how long one should simulate $X(t)-c t$ in order to get the assumed accuracy in estimation of $\mathbb{P}(Q>u)$. 
Let

$$
\gamma(u, t):=\mathbb{P}(Q>u)-\mathbb{P}\left(Q_{\mathrm{tr}}(t)>u\right) .
$$

Notice that

$$
\gamma(u, t)=\mathbb{P}\left(\sup _{s \in[0, t]}(X(s)-c s) \leq u ; \sup _{s \in(t, \infty)}(X(s)-c s)>u\right) .
$$

Apart from the case of Brownian input, for which

$$
\gamma(u, t)=\mathrm{e}^{-2 u c} \mathbb{P}(\mathcal{N}>(c t-u) / \sqrt{t})-\mathbb{P}(\mathcal{N}>(c t+u) / \sqrt{t}),
$$

where $\mathcal{N}$ is the standard normal random variable, little is known about the behavior of $\gamma(u, t)$ for general Gaussian inputs.

Open Problem 3.1 Find the asymptotics of $\gamma(u, t)$, as $t \rightarrow \infty$, for fixed $u$.

This setting is intimately related with the notion of relaxation time, which was intensively investigated in the classical, non-Gaussian queueing context.

Recent progress in understanding the behavior of $\gamma(u, t)$ was made in [18], where

$$
\mathbb{D}_{1}(t):=\sup _{u>0} \gamma(u, t), \quad \text { and } \quad \mathbb{D}_{2}(t):=\int_{0}^{\infty} \gamma(u, t) \mathrm{d} u
$$

were studied for queues driven by fractional Brownian motion. Interestingly, in [18] it was proved that

$$
\lim _{t \rightarrow \infty} \frac{\log \mathbb{D}_{1}(t)}{t^{2-2 H}}=\lim _{t \rightarrow \infty} \frac{\log \mathbb{D}_{2}(t)}{t^{2-2 H}}=\lim _{t \rightarrow \infty} \frac{\log \mathbb{P}(K>t)}{t^{2-2 H}},
$$

where

$$
K:=\inf \{s \geq 0: Q(s)=0\}-\sup \{s \leq 0: Q(s)=0\}
$$

denotes the ongoing busy period at time 0 ; the rightmost decay rate in (4) was computed in [17]. We expect that this result carries over to the class of Gaussian inputs with regularly varying variance function at $\infty$. In view of (4), the following challenging question arises.

Open Problem 3.2 Find the exact asymptotics of metrics $\mathbb{D}_{1}(t), \mathbb{D}_{2}(t)$, as well as those of $\mathbb{P}(K>t)$, as $t \rightarrow \infty$.

\section{Estimates and simulation of the asymptotic constant}

Over the past decade, the asymptotic behavior of $\mathbb{P}(Q>u)$, as $u \rightarrow \infty$, was an important research theme, both for FBM and IG driven queues; see [3, 10, 13, 14]. The structural form of these asymptotics is known now, and captured by the following general formula:

$$
\mathbb{P}(Q>u)=\mathcal{C} u^{\beta} \Psi(m(u))(1+o(1)),
$$


as $u \rightarrow \infty$, with known $\beta>0$,

$$
m(u):=\min _{t \geq 0} \frac{u+c t}{\sigma_{X}(t)},
$$

and $\Psi(u):=\mathbb{P}(\mathcal{N}>u)$. The asymptotic constant $\mathcal{C}$ in (5) can be expressed in terms of the so-called generalized Pickands constant $\mathcal{H}_{\eta}$, associated with a Gaussian process $\eta(t)$ that directly relates to our input process on $X(t)$. Generally, neither an explicit formula nor an accurate approximation for $\mathcal{C}$ is known.

Recall that by generalized Pickands constant $\mathcal{H}_{\eta}$ we understand the limit [3]

$$
\lim _{T \rightarrow \infty} \frac{\mathcal{H}_{\eta}(T)}{T}=\mathcal{H}_{\eta}
$$

where $\mathcal{H}_{\eta}(T):=\mathbb{E} \exp \left(\max _{t \in[0, T]}\left(\sqrt{2} \eta(t)-\sigma_{\eta}^{2}(t)\right)\right)$. In order to ensure that $\mathcal{H}_{\eta}$ is well defined, it is assumed that $\eta(t)$ is a centered Gaussian process with stationary increments, a.s. continuous sample paths, $\eta(0)=0$ and such that the variance function $\sigma_{\eta}^{2}(t)$ satisfies

C1 $\sigma_{\eta}^{2}(t) \in C^{1}([0, \infty))$ is strictly increasing and there exists $\epsilon>0$ such that $\lim \sup _{t \rightarrow \infty} t \dot{\sigma}_{\eta}^{2}(t) / \sigma_{\eta}^{2}(t) \leq \epsilon$

C2 $\sigma_{\eta}^{2}(t)$ is regularly varying at 0 with index $\alpha_{0} \in(0,2]$ and $\sigma_{\eta}^{2}(t)$ is regularly varying at $\infty$ with index $\alpha_{\infty} \in(0,2)$.

We note that, for models with $\{X(t): t \in \mathbb{R}\}$ having a regularly varying variance function at $\infty$ with $\alpha_{\infty}>1$, the asymptotic constant $\mathcal{C}$ reduces to the classical Pickands constant $\mathcal{H}_{B_{H}}$ with $H=\alpha_{\infty} / 2$; see $[10,13]$.

The constant $\mathcal{H}_{B_{H}}$ has been widely studied, but just a few partial results have been obtained so far. In particular, the exact value of $\mathcal{H}_{B_{H}}$ is known only for $\mathcal{H}_{B_{1 / 2}}=1$ and $\mathcal{H}_{B_{1}}=1 / \sqrt{\pi}$; see [22]. Some estimates for $\mathcal{H}_{B_{H}}$ are given in [3, 4, 8, 25], but the gap between the lower and upper bounds is still quite substantial. For example, bounds for $\mathcal{H}_{B_{H}}$ are precise only in the neighborhood of $H=1 / 2$ and $H=1$; see [4]. This makes the following open problem to be particularly important.

Open Problem 4.1 Find further characterizations of $\mathcal{H}_{\eta}$ that lead to more precise estimates of (6).

The following conjecture should perhaps be seen as mathematical folklore, as it lacks any firm heuristic support. However, due to the lack of precision of currently known estimates, is has not been falsified so far.

Conjecture 4.1 $\mathcal{H}_{B_{H}}=1 / \Gamma\left(\frac{1}{2 H}\right)$.

Obtaining exact values for $\mathcal{H}_{\eta}$ being prohibitively hard, it is of great importance to develop stable algorithms for estimating Pickands constants by simulation. It is evident that methods based on the definition (6) cannot produce any efficient algorithms. This is not only due to the fact that

$$
\exp \left(\max _{t \in[0, T]}\left(\sqrt{2} \eta(t)-\sigma_{\eta}^{2}(t)\right)\right)
$$


is asymptotically lognormal, but also a consequence of the fact that for each $\varepsilon>0$

$$
\lim _{T \rightarrow \infty} \frac{1}{T} \mathbb{E} \exp \left(\max _{t \in[0, T]}\left(\sqrt{2} \eta(t)-(1+\varepsilon) \sigma_{\eta}^{2}(t)\right)\right)=0,
$$

while

$$
\lim _{T \rightarrow \infty} \frac{1}{T} \mathbb{E} \exp \left(\max _{t \in[0, T]}\left(\sqrt{2} \eta(t)-(1-\varepsilon) \sigma_{\eta}^{2}(t)\right)\right)=\infty .
$$

This explains why the methods needed cannot be straightforward, crude Monte Carlo type of procedures. There is some hope of developing a reliable simulation method based on the so-called change of measure technique. For the very special case of $\eta(t)=\int_{0}^{t} Z(s) \mathrm{d} s$, where $Z(t)$ is an Ornstein-Uhlenbeck process, this approach produced a stable algorithm; see [8]. However, applying a somewhat similar technique to estimate $\mathcal{H}_{B_{H}}$, resulted in two completely different estimates: compare [2] with [19].

Open Problem 4.2 Develop a reliable simulation algorithm for efficient estimation of $\mathcal{H}_{\eta}$.

Finding an efficient technique to estimate $\mathcal{H}_{\eta}$ by simulation is a challenging task which is important not only from the perspective of the theory Gaussian fluid queues, but also in light of the theory of extreme values of stochastic processes in a more general sense.

Acknowledgements K.D. was supported by MNiSW Grant N N201 394137 (2009-2011). K.D. and M.M. thank the Isaac Newton Institute, Cambridge, for hospitality.

Open Access This article is distributed under the terms of the Creative Commons Attribution Noncommercial License which permits any noncommercial use, distribution, and reproduction in any medium, provided the original author(s) and source are credited.

\section{References}

1. Abate, J., Whitt, W.: The correlation function of RBM and $M / M / 1$. Stoch. Models 4, 315-359 (1988)

2. Burnecki, Z., Michna, Z.: Simulation of Pickands constants. Probab. Math. Stat. 22, 193-199 (2002)

3. Dȩbicki, K.: Ruin probabilities for Gaussian integrated processes. Stoch. Process. Appl. 98, 151-174 (2002)

4. Dȩbicki, K., Kisowski, P.: A note on upper estimates for Pickands constants. Stat. Probab. Lett. 78, 2046-2051 (2008)

5. Dȩbicki, K., Mandjes, M.: Traffic with an FBM limit: a convergence result. Queueing Syst. 46, 113127 (2004)

6. Dȩbicki, K., Palmowski, Z.: Heavy traffic asymptotics of on-off fluid model. Queueing Syst. 33, 327338 (1999)

7. Dȩbicki, K., Rolski, T.: Gaussian fluid models; a survey. In: Symposium on Performance Models for Information Communication Networks, Sendai, Japan, 23-25 January 1999 (2000)

8. Dȩbicki, K., Michna, Z., Rolski, T.: Simulation of the asymptotic constant in some fluid models. Stoch. Models 19, 407-423 (2003)

9. Dȩbicki, K., Es-Saghouani, A., Mandjes, M.: Transient characteristic of Gaussian fluid queues. Queueing Syst. 62, 383-409 (2009)

10. Dieker, A.B.: Extremes of Gaussian processes over an infinite horizon. Stoch. Process. Appl. 115, 207-248 (2005) 
11. Es-Saghouani, A., Mandjes, M.: On the correlation structure of Gaussian queues. Stoch. Models 25, 221-247 (2009)

12. Glynn, P., Mandjes, M.: Simulation-based computation of the workload correlation function in a Lévydriven queue. CWI Report PNA-E0911 (2009)

13. Hüsler, J., Piterbarg, V.: Extremes of a certain class of Gaussian processes. Stoch. Process. Appl. 83, 257-271 (1999)

14. Hüsler, J., Piterbarg, V.: On the ruin probability for physical fractional Brownian motion. Stoch. Process. Appl. 113, 315-332 (2004)

15. Kulkarni, V., Rolski, T.: Fluid model driven by an Ornstein-Uhlenbeck process. Probab. Eng. Inf. Sci. 8, 403-417 (1994)

16. Mandjes, M.: Large Deviations for Gaussian Queues. Wiley, Chichester (2007)

17. Mandjes, M., Mannersalo, P., Norros, I., van Uitert, M.: Large deviations of infinite intersections of events in Gaussian processes. Stoch. Process. Appl. 116, 1269-1293 (2006)

18. Mandjes, M., Norros, I., Glynn, P.: On convergence to stationarity of fractional Brownian storage. Ann. Appl. Probab. 19, 1385-1403 (2009)

19. Michna, Z.: On tail probabilities and first passage times for fractional Brownian motion. Math. Methods Oper. Res. 49, 335-354 (1999)

20. Mikosch, T., Resnick, S., Rootzén, H., Stegeman, A.: Is network traffic approximated by stable Lévy motion or fractional Brownian motion. Ann. Appl. Probab. 12, 23-68 (2002)

21. Norros, I.: A storage model with self-similar input. Queueing Syst. 16, 387-396 (1994)

22. Piterbarg, V.I.: Asymptotic Methods in the Theory of Gaussian Processes and Fields. Translations of Mathematical Monographs, vol. 148. AMS, Providence (1996)

23. Reich, E.: On the integrodifferential equation of Takács I. Ann. Math. Stat. 29, 563-570 (1958)

24. Reynolds, J.: The covariance structure of queues and related processes-a survey of recent work. Adv. Appl. Probab. 7, 383-415 (1975)

25. Shao, Q.M.: Bounds and estimators of a basic constant in extreme value theory of Gaussian processes. Stat. Sin. 6, 245-257 (1996)

26. Taqqu, M.S., Willinger, W., Sherman, R.: Proof of a fundamental result in self-similar traffic modeling. Comput. Commun. Rev. 27, 5-23 (1997) 